\title{
8.7 PULSAR (AND X-STAR) EMISSION FROM THE MAGNETOSPHERE OF A COLLAPSED STAR
}

\author{
BRUNO COPPI \\ Massachusetts Institute of Technology, Cambridge, Mass., U.S.A. \\ and \\ ATTILIO FERRARI \\ Laboratorio di Cosmo-geofisica del CNR and Istituto di Fisica dell'Università, Torino, Italy
}

\begin{abstract}
We propose nonthermal plasma mechanisms to account for the most evident physical characteristics of pulsar emission, including the production of $\mathrm{X}$ - and $\gamma$-rays and the acceleration of high-energy particles. Special reference to the Crab Nebula pulsar (NP 0532) is made and an application of the same model to other non-thermal X-ray sources is suggested.
\end{abstract}

\section{Quasi-Equilibrium of the Plasma Magnetosphere}

Following the arguments proposed by several authors (Gold, 1968; Large et al., 1968), we assume that the condensed stars associated with pulsars are strongly magnetized and rapidly rotating as a consequence of a gravitational collapse and of angular momentum and magnetic flux conservation. It becomes then natural to assume that the star is surrounded (inside the 'speed of light' cylinder $R_{c}=c / \omega_{0}, \omega_{0}$ being the angular velocity of rotation) by a relatively dense plasma with a small charge separation since, if this were not the case, unrealistically large electric fields (of the order of $u_{0} B, u_{0}$ being the velocity required for co-rotation and $B$ the magnetic induction) would develop along the magnetic field lines (Goldreich and Julian, 1969).

As a consequence of the high value of the magnetic field $B\left(B_{\text {surf }} \gtrsim 10^{10} \mathrm{G}\right)$, the Langmuir frequency $\omega_{p e}$ is much smaller than the electron gyrofrequency $\Omega_{e}$. Taking $B_{0}=10^{10} \mathrm{G}$ and $n_{0}=10^{18} \mathrm{~cm}^{-3}$ as reference values, we have in fact $\Omega_{e} / \omega_{p e}=3.1 \times 10^{3}$ $\left(B / B_{0}\right)\left(n_{0} / n_{e}\right)^{1 / 2}$.

In these conditions, considering, for instance, mildly relativistic electrons with energy perpendicular to the magnetic field, they will tend to lose it by synchrotron radiation in a typical time $\tau \approx 2.58 \times 10^{-12}\left(B_{0} / B\right)^{2} \mathrm{sec}$. As a consequence of radiation losses, these electrons have a tendency to acquire an anisotropic distribution function in velocity space, with more particles having velocity parallel to the magnetic field than perpendicular. In this case the plasma is subject to microinstabilities which tend to restore the distribution function isotropy.

Thus, by taking into account the effects of collective phenomena (such as microinstabilities) and interparticle collisions, we can assume that the plasma surrounding a strongly magnetized collapsed star is in macroscopic quasiequilibrium. A stationary state of this type, in the presence of fluctuations excited by the electron distribution function anisotropy in a strong magnetic field, may be described by the so-called quasilinear approximation (Shapiro and Shevchenko, 1968). The possibility of an 
equilibrium (Chandrasekhar, 1961) is particularly important for cases in which the magnetic configuration is not symmetric around the star's axis of rotation, and which are suitable for pulsar models (e.g. Gold, 1968; Eastlund, 1968). The lack of rotational symmetry is, in fact, indicated by the observation of pulses and the presence of an 'equilibrium' by the remarkably stable pulse pattern.

Assuming, for simplicity, that the plasma under consideration is composed of electrons and ions of charge $Z$, the equilibrium of the plasma region inside the 'speed of light' cylinder can be approximately represented by a set of proper moment equations. In particular the electron-momentum balance equation can be written as

$$
\begin{aligned}
\mathbf{E}^{\prime} & =-\mathbf{u}_{e}^{\prime} \times \frac{\mathbf{B}}{c}-\frac{m_{e}}{e}\left[\mathfrak{f}_{e} \cdot\left(\mathbf{u}_{e}^{\prime}-\mathbf{u}_{i}^{\prime} \frac{Z n_{i}}{n_{e}}\right)\right. \\
& \left.+\frac{1}{m_{e} n_{e}}\left(\nabla \cdot \mathfrak{P}_{e}-\mathbf{S}_{e}^{(p)}\right)+\mathbf{u}_{e}^{\prime} \cdot \nabla \mathbf{u}_{e}^{\prime}+2 \omega_{0} \times \mathbf{u}_{e}^{\prime}-\mathbf{g}\right] .
\end{aligned}
$$

Here primed quantities refer to the frame co-rotating with the star; $\mathbf{g}$ indicates gravity, $n$ density, $\mathbf{u}$ average velocity and subscripts $e$ and $i$ refer to electrons and ions. $\mathbf{S}_{\boldsymbol{e}}^{(p)}$ represents the momentum source that must be taken into account if, for instance, particle pair creation by high energy photons occurs. The tensor $\mathfrak{f}_{e}$ is an 'effective' collision frequency, representing the momentum exchange between electrons and ions, and $\mathfrak{P}_{e}$ is the relativistic electron-pressure tensor. Both of these tensors incorporate the effects of particle collisions, collective modes and kinetic processes involving detailed consideration of velocity space, that is knowledge of the electron distribution function $f_{e}(\mathbf{r}, \mathbf{v})$. A similar equation can be derived for the ions and by properly summing the two, one obtains the total momentum conservation equation and, consequently, the small current density $\mathbf{J}_{\perp}^{\prime}$ required to maintain the equilibrium transverse to the magnetic field

$$
\begin{aligned}
\mathbf{J}_{\perp}^{\prime} & =\left(\mathbf{B} / B^{2}\right) \times\left\{e \mathbf{E}^{\prime}\left(n_{e}-Z n_{i}\right)+\sum_{j=i, e} m_{j} n_{j}\right. \\
& \left.\times\left(\mathbf{u}_{j}^{\prime} \cdot \nabla \mathbf{u}_{j}^{\prime}+2 \omega_{0} \times \mathbf{u}_{j}^{\prime}-\mathbf{g}\right)+\nabla \cdot \mathfrak{P}_{j}-\mathbf{S}_{j}^{(p)}\right\} .
\end{aligned}
$$

The equilibrium parallel to $\mathbf{B}$ can be described by the $\mathbf{B} / \mathbf{B}$ component of Equation (1), which as usually done in plasma physics, can be written as

$$
E_{\|}^{\prime}=\eta_{\text {an }} J_{\|}^{\prime}
$$

$J_{\|}^{\prime}$ being the longitudinal current density and $\eta_{\text {an }}$ the anomalous, or nonclassical resistivity that takes into account all relevant collisional and turbulent plasma processes (Kadomtsev and Pogutze, 1968; Coppi and Mazzucato, 1969). We recall, in fact, that in many significant cases $\eta_{\text {an }}$ has been found experimentally (Artsimovich et al., 1968) to be orders of magnitude higher than the classical resistivity based on the momentum exchange in electron-proton collisions (see Figure 1). 


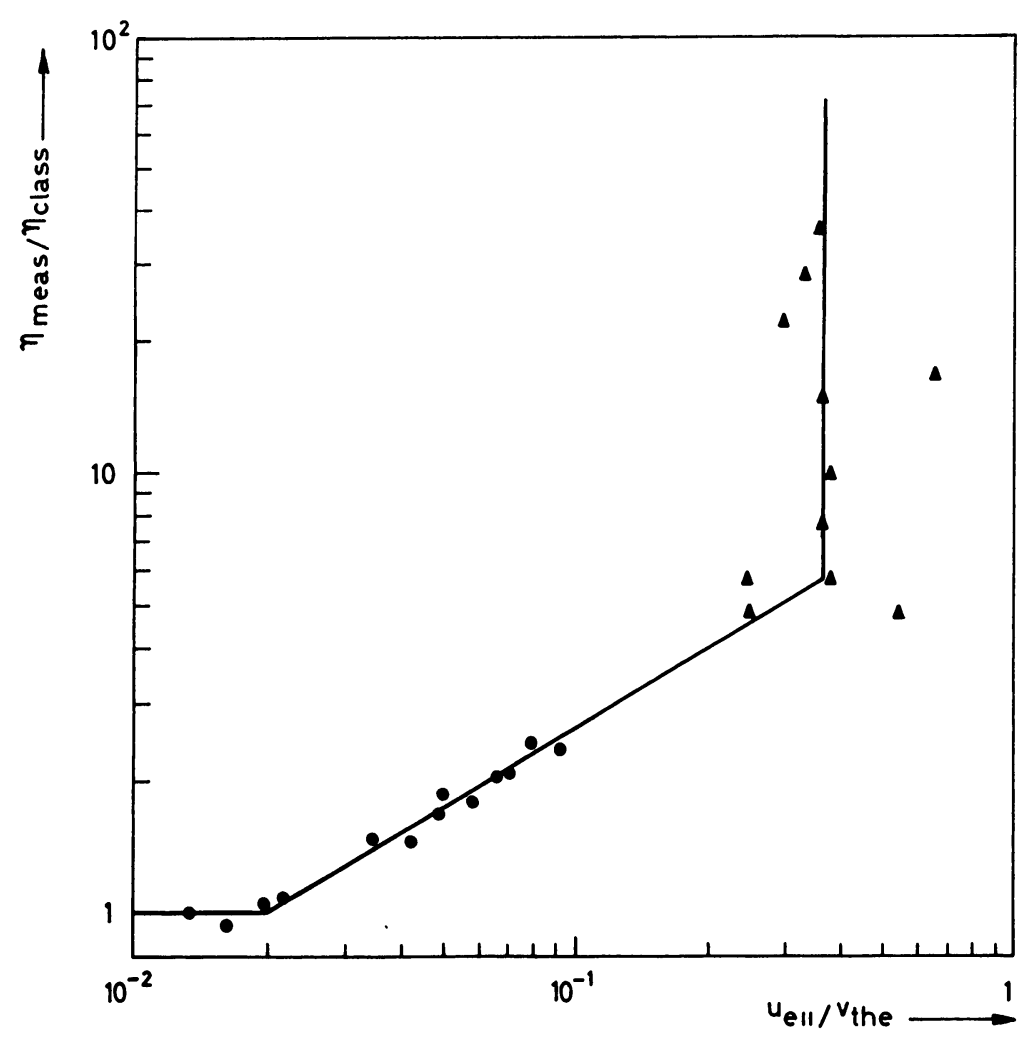

Fig. 1. Ratio of the measured plasma resistivity ( $\left.\eta_{\text {meas }}\right)$ to the theoretical one ( $\left.\eta_{\text {class }}\right)$ evaluated from the effects of interparticle collisions only. The experimental points were obtained from data on plasmas confined in strong magnetic field with $u_{e \|}$ being the electron flow velocity along the magnetic field and $v_{\text {the }}$ the electron thermal velocity. See Coppi and Mazzucato (1969).

The relationship between $J_{\|}^{\prime}$ and $\mathbf{J}_{\perp}^{\prime}$ is given by the charge conservation equation

$$
\nabla \cdot \mathbf{J}^{\prime}=0
$$

Here we have to assume that $\mathbf{J}^{\prime} \simeq \alpha \mathbf{B}$, to lowest order in order not to have unrealistic forces $\mathbf{J}^{\prime} \times \mathbf{B}$. Poisson's equation reads

$$
\nabla \cdot \mathbf{E}^{\prime}=4 \pi e\left(Z n_{i}-n_{e}\right)+\nabla \cdot\left(\mathbf{u}_{0} \times \frac{\mathbf{B}}{c}\right),
$$

where $\mathbf{u}_{0}=\omega_{0} \times \mathbf{r}$. Notice that the electric field seen in the inertial frame is

$$
\mathbf{E}=\mathbf{E}^{\prime}-\mathbf{u}_{0} \times \mathbf{B} / c .
$$

Therefore, the plasma could be in exact co-rotation (Chandrasekhar, 1961; Goldreich and Julian, 1969) with the star magnetic field only if $E_{\|}^{\prime}=0$, a circumstance that is 
clearly not possible since, as we have seen, collective, collisional and inertial effects are necessary to the existence of the plasma quasi-equilibrium.

Noting that the field:

$$
\left|\mathbf{u}_{0} \times \mathbf{B} / c\right| \simeq 1.2 \times 10^{10}\left[\omega_{0} B r /\left(\omega^{0} B_{0} r_{0}\right)\right] \mathrm{V} / \mathrm{cm}
$$

where $r_{0}=2 \times 10^{6} \mathrm{~cm}$ and $\omega^{0}=2 \times 10^{2} \mathrm{rad} / \mathrm{sec}$ are reference values applicable to the Crab Nebula pulsar, we argue that a very small lag between particles and magnetic field exists corresponding to $\mathbf{E}^{\prime}$, and in particular $E_{\|}^{\prime}$ being non zero although much less than $\left|\mathbf{u}_{0} \times \mathbf{B} / c\right|$.

Very small values of $E^{\prime} / E_{\perp}$ which for $n_{e} \approx n_{0}$ correspond to a relative charge separation $1-Z n_{i} / n_{e} \approx 10^{-7}$ as indicated by Equation (5), are consistent with a prevalence of the electromagnetic force terms in Equation (1) and in the total momentum conservation equation. Therefore, we can concentrate on looking at the plasma aspect of the problem and consider, in more detail, the equilibrium along a typical open line of force emanating from a pole or a spot of the star.

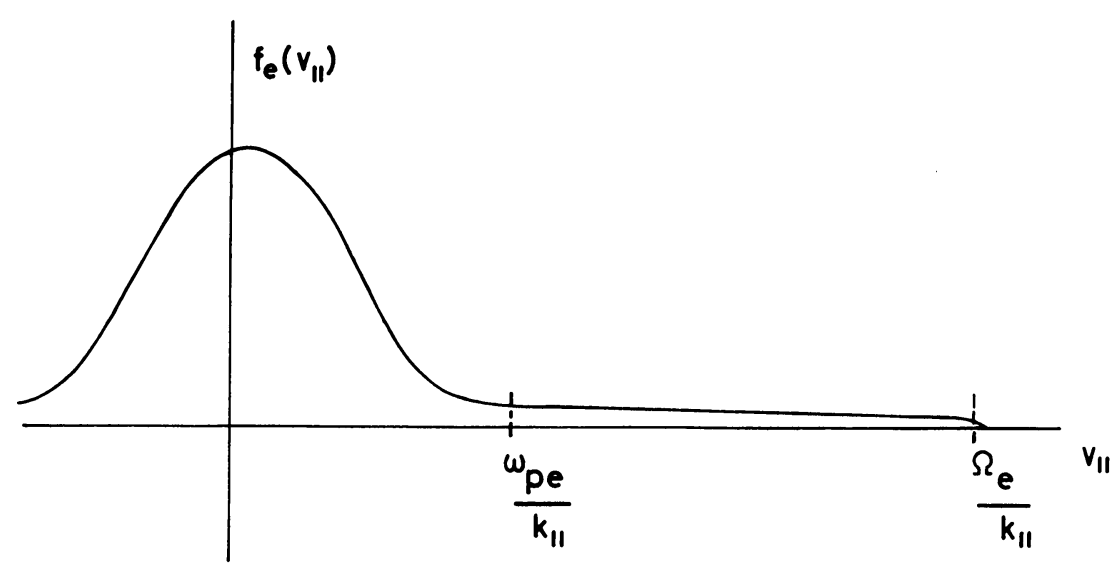

Fig. 2. Example of a typical 'runaway' distribution, with regions of particle-wave resonance indicated.

Some points have to be emphasized in relation with the existence of this equilibrium.

(1) An important limit for $E_{\|}^{\prime}$ is the runaway critical field $E_{\text {run }}=v_{e i} m_{e} v_{\text {the }} / e$, where $v_{e i}$ is the average classical electron-ion collision frequency and $v_{\text {the }}$ the electron thermal velocity. The ratio $E_{\|}^{\prime} / E_{\text {run }}$ is, in fact, significant in relation to the tail of superthermal electrons that characterizes the electron distribution runction $f_{e}\left(v_{\|}\right), \dot{v}_{\|}$being the electron velocity along $B$. In other words, for not-too-low values of $E_{\|}^{\prime} / E_{\text {run }}$ the typical profile of $f_{e}\left(v_{\|}\right)$that results as a consequence of electron collisions and collective modes is of the type represented in Figure 2. We recall for this that in magnetically confined plasmas (Stodiek, 1969) with temperatures $1 \lesssim T_{e} \lesssim 10 \mathrm{eV}$, electrons of about $200 \mathrm{keV}$ with density $\approx 10^{-2} n_{e}$ have been observed for $10^{-1}<E_{\|} / E_{\text {run }}<1$. 


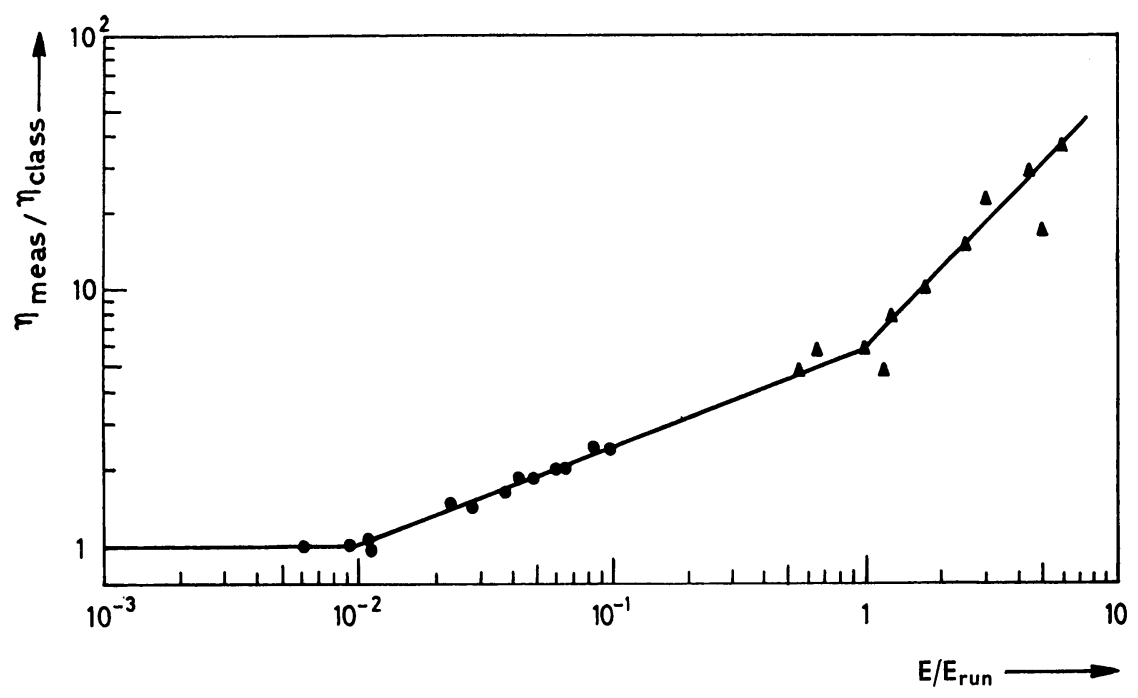

Fig. 3 The same ratio $\eta_{\text {meas }} / \eta_{\text {class }}$ as in Figure 1 versus $E_{\|} / E_{\text {run }}, E_{\|}$being the electric field parallel to the magnetic field and $E_{\text {run }}$ the critical 'runaway' field.

Moreover, for plasmas with $\omega_{p e}<\Omega_{e}$, the anomalous resistivity $\eta_{\mathrm{an}}$ has been found to become much larger than $\eta_{c l}=v_{e i} m_{e} /\left(n_{e} e^{2}\right)$ for $E_{\|} \gtrsim E_{\text {run }}$ (see Figure 3). A numerical evaluation of the runaway field is

$$
E_{\text {run }} \simeq 3.1 \times 10^{4} \alpha n_{e} T_{0} /\left(n_{0} T_{e}\right) \mathrm{V} / \mathrm{cm},
$$

where $T_{0}=10^{6} \mathrm{~K}$ has been taken as typical electron temperature in the vicinity of the star following current neutron star models, and $\alpha=1-0.13 \log _{10}\left(n_{e} / n_{0}\right)+$ $0.26 \log _{10}\left(T_{e} / T_{0}\right)$.

(2) The longitudinal energy gained by the electrons under the influence of $E_{\|}^{\prime}$ is scattered in the transverse (to B) direction by electron-proton collisions or by collective effects such as the following (e.g. Coppi et al., 1969). A plasma electrostatic (longitudinal) wave with frequency $\omega=\omega_{p e}\left(k_{\|} / k\right), \mathbf{k}$ being the wave vector, is involved in particle-wave resonances producing the energy and momentum exchange

$$
\hbar \omega+\Delta \varepsilon_{j}=0, \quad \hbar \mathbf{k}+\Delta \mathbf{p}_{j}=0
$$

where $\Delta \varepsilon_{j}$ is the energy variation and $\Delta \mathbf{p}_{j}$ the momentum variation for particles of species $j$, while $\hbar \omega$ and $\hbar \mathbf{k}$ refer to the wave. Now, $\Delta \varepsilon_{j}=\Delta \varepsilon_{\| j}+\Delta \varepsilon_{\perp j}$, with $\Delta \varepsilon_{\| j}=$ $=m_{j} v_{\|} \Delta v_{\|}, \Delta \varepsilon_{\perp j}=n^{0} \hbar \Omega_{j}, n^{0}$ being an integer. In addition, $\Delta p_{\| j}=m_{j} \Delta v_{\|}$, while $\Delta p_{\perp j}$ is taken up by the magnetic field. Therefore, the wave-electron resonance condition is:

$$
\omega+n^{0} \Omega_{e}-k_{\|} v_{\|}=0 .
$$

Now, since $\omega \ll \Omega_{e}$, Equation (10) shows that the energy exchanged with the wave is negligible for $n^{0} \neq 0$, and the main effect is a transfer of longitudinal electron energy into transverse energy. Notice that a plasma wave of the considered frequency can 
exist, without being strongly damped, only for $\omega \gg k_{\|} v_{\text {the }}$. Therefore, the electrons participating in the resonance (10) have to be highly energetic and belong to the distribution tail. In particular, the relevant theory requires that $\left(\partial f_{e} / \partial v_{\perp}\right)_{v \|}=\Omega_{e} / k_{\|}$be sufficiently large for the process represented by Equation (10) not to be overcome by the ordinary Landau damping corresponding to $n^{0}=0$ and involving particles with $v_{\|}=\omega / k_{\|} \ll \Omega_{e} / k_{\|}$(Figure 2). Considering the high values that $\Omega_{e} / \omega_{p e}$ can have for the case under consideration, it is clear that the resonating particles have to be relativistic and in this case Equation (10) becomes roughly $\gamma k_{\|} c \approx \Omega_{e 0}$, where $\Omega_{e 0}$ is the rest mass gyrofrequency.

(3) The observed high anomalous resistivity in laboratory plasma experiments with $\omega_{p e}<\Omega_{e}$ is attributed to wave-particle interactions of the type discussed above or of other types associated, for instance, with streaming instabilities. Therefore, a large $E_{\|}$can be compatible with acceptable values of $J_{\|}$.

(4) The current $J_{\|}$can also excite low-frequency modes with $\omega<\omega_{p e}$ and $\omega<\Omega_{i}$, $\Omega_{i}$ being the ion gyrofrequency. For this we may consider, among other possibilities, modes of the ion-sound wave type, which depend on $T_{e} \neq 0$, have a frequency $\omega \approx k_{\|} \sqrt{ }\left(T_{e} / m_{p}\right)$, are electrostatic and are associated with finite electron thermal conductivity (Coppi and Mazzucato, 1969) or with electron Landau damping along the same direction.

Finally we remark that the driving electric field can be related to the strong slippage of the plasma with respect to the rotating magnetic field in the far out regions (Goldreich and Julian, 1969), and is induced by the star rotation. So this is the ultimate energy source for all the emission processes.

\section{Plasma Radiation Mechanisms}

From the discussion given above it follows that two main classes of emission processes can be considered. One is related to the existence of a sizeable tail of superthermal electrons and therefore to not-too-low values of $E_{\|} / E_{\text {run }}$. Another is associated with the main body of the electron distribution and can be effective at very low values of $E_{\|} / E_{\text {run }}\left[\right.$ for instance $E_{\|} / E_{\text {run }} \approx\left(m_{e} / m_{p}\right)^{1 / 2}$ ].

We recall for this that the appearance of 'runaway' electrons in a collisional nonturbulent plasma can be represented by a sharp function of $E_{\|} / E_{\text {run }}$, typically of the form (Gurevich, 1961)

$$
n_{s} \approx n_{e} e^{-E_{\text {run }} / E_{11}} .
$$

Then, considering the first class of processes, we notice that:

(a) X-ray emission by mildly relativistic electrons (with $\gamma=\varepsilon / m c^{2} \simeq 1$ ) is related to the basic role of the frequency $\Omega_{e}$ in plasma collective modes. This emission can, in fact, be the result of direct cyclotron radiation by those electrons that have acquired transverse energy by a scattering process.

(b) $\gamma$-ray emission, possibly due to a scattering process similar to that for X-ray emission can result from synchrotron radiation by highly relativistic electrons. 
(c) Optical emission is expected to follow along with $\mathrm{X}$-ray emission. For instance if longitudinal high frequency plasma modes are excited, a frequency decay process toward lower frequencies and coupling to electromagnetic modes take place (Kaplan and Tsytovich, 1969).

(d) A nonlinear frequency decay process can also contribute to the infrared and upper band of the radio spectrum. In addition, a direct coupling of the longitudinal waves (having $\omega \approx k_{\|} \omega_{p e} / k$ and owing their excitation to the anisotropy of the tail of superthermal electrons) with transverse waves can be relevant to this range or even to the optical, if $n_{e} \approx n_{0}$.

(e) A small fraction of the total electron population accelerated by $E_{\|}$will be able to escape all collisional and collective interactions and to attain very high energies in the direction of the magnetic field, so that a process for direct particle acceleration is also to be considered.

(f) In reference to Equation (11) we may argue that a collapsed star, because of its initial conditions and of aging which implies slowing-down and probably decreasing $T_{e}$, can reach a point where the ratio $E_{\|} / E_{\text {run }}$ is no longer sufficient to maintain an adequate population of fast particles to make the particle wave resonance with $n^{0} \neq 0$ prevail over ordinary Landau damping. This corresponds to the resonance $\omega-k_{\|} v_{\|}=0 \quad\left(n^{0}=0\right)$, which generally involves a considerably larger number of particles (Fig. 2).

Referring to the second class of emission processes, we consider the low-frequency longitudinal modes that are excited by $J_{\|}$. These modes, after propagating outward along the magnetic field lines, can couple (in regions of lower density) to longitudinal plasma waves with frequency $\sim \omega_{p e}$, and then with transverse waves (Ginzburg and Zaitsev, 1969) (in the radio band) that are expected to be strongly polarized. So this emission mechanism is characterized by propagation direction clearly related to that of the magnetic field, and does not exclude the possibility that the emitted wave be linearly or circularly polarized.

\section{Application of Plasma Model to Pulsars}

A plasma model of the type given above can be applied more specifically to the interpretation of the Crab Nebula pulsar emission and to other pulsars and X-ray sources. Then, on the basis of the physical processes discussed in the previous section, we will divide the pulsar spectrum in high-energy and low-energy regions in agreement with observational evidence from NP 0532. Neugebauer et al. (1969) have in fact measured a break-down of the spectrum in the infrared region, so that two distinct peaks appear in the radio region and in the $\mathbf{X}$ (or possibly $\gamma$ ) region.

\section{A. HIGH-ENERGY EMISSION}

The Crab Nebula pulsar, because of its large angular velocity and of its recent formation can be assumed to have an electron parallel distribution function with a sizeable tail of superthermal electrons. We notice that the corresponding lack of 
isorotation of the plasma with the star's magnetic field leads to a reasonably small variation of the rotational velocity over the scale length of the fields.

We also recall from the previous sections that runaway electrons with high parallel energy can undergo pitch-angle scattering or by plasma collective modes and then acquire perpendicular energy. This will be lost by cyclotron radiation at the harmonics of the electron cyclotron frequency $\Omega_{e}=1.76 \times 10^{17} \gamma^{-1}\left(B / B_{0}\right)(\gamma$ being the relativistic factor, $\left.\gamma=\varepsilon / m_{0} c^{2}\right)$, which for mildly relativistic electrons is in the X-range.

As pointed out earlier, we consider the optical emission as strictly correlated with the higher energy emission either by means of a decay process of higher frequency modes or by direct coupling of Langmuir waves with electromagnetic plasma modes. The efficiency of the decay process does not have to be high, since the observations for the Crab pulsar give $W_{\text {opt }} / W_{x} \approx 10^{-3}$. As for the direct coupling, we notice that the plasma waves giving electron pitch-angle scattering have frequency $\omega=\left(k_{\|} / k\right) \omega_{p e}$; so, if the density is sufficiently high $\left(n_{e} \gtrsim n_{0}\right)$, optical emission is produced and this is evidently correlated with X-rays.

$\gamma$-ray emission, which has been tentatively identified recently (Vasseur et al., 1970; Kinzer et al., 1971; Hillier et al., 1971) obviously involves highly relativistic particles. So one possibility is that these particles emit by synchrotron radiation and this be maintained by pitch-angle scattering processes. Another possibility comes from considering (see previous sections) the relatively small population of runaway electrons which can escape all the effects of interparticle collisions and collective phenomena. So, $\gamma$-rays can be thought of as being originated by the single particle acceleration process along open field lines emanating from poles or spots not aligned with the rotation axis.

\section{B. LOW-ENERGY EMISSION}

We refer, for the radio emission, to low-frequency modes which can be excited by the current $J_{\|}$as indicated in the previous section.

These longitudinal waves do not require the presence of runaway electrons. They can propagate towards outer regions of decreasing density where they can couple with Langmuir waves and electromagnetic plasma waves propagating transversely to the magnetic field. In these conditions it is not difficult to reconcile propagation along the magnetic field of waves with frequency $\omega<\omega_{p e}$ with the observed linear polarization for the Crab pulsar and several others. In particular, we expect that the lower the frequency the further is the distance to which the electrostatic low-frequency modes have to propagate before coupling to electromagnetic modes. Since the beam of open lines of force widens with increasing distance from the star pole or spot, the process we have proposed may contribute to the observed progressive pulse wideing as the (radio) frequency decreases (Drake, 1969).

On the subject of linear polarization we recall that the optical pulses from NP 0532 (Crab), as the radio pulses from PSR 0833 (Vela) exhibit a wide rotation of the angle polarization within the pulse (Wampler et al., 1969). We may explain this observation assuming that in these cases the coupling of electrostatic modes with electromagnetic 
waves occurs close to the star where the magnetic field changes direction over a relatively short distance. Thus the radiation that is received at each instant within the pulse may come from a sequence of different points along a given magnetic tube of flux, where the magnetic field direction undergoes a finite change (such as for instance, $60^{\circ}$ ). The electromagnetic modes which propagate perpendicularly to this direction have their electric field strongly correlated to it and reflect its variation.

We also recall that the single radio pulses from several pulsars have been observed to have a large circularly polarized component (Smith, 1970) with equal probability of left hand and right hand polarization. In these cases part of the received wave energy should be carried along the received star's magnetic field lines by plasma electromagnetic waves which, as is well known, are circularly polarized in either direction.

Thus far, NP 0532 is the only observed pulsar emitting over a wide range of frequencies. Attempts to detect optical or X-emission from other pulsars have failed. On the other hand, the period of PSR 0833 (Vela) is less than three times longer than that of NP 0532 and yet PSR 0833 exhibits only radio emission. So, although there is no reason to believe that the two pulsars were born with the same physical characteristics, the possibility of a sharp aging process, with a short $\mathrm{X}$ and optical phase followed by a relatively long radio-emission phase, has to be considered. For this we recall that X-ray and optical emission was related to the existence of a sizeable tail of superthermal electrons, and that the appearance of this tail is represented by a sharp function of $E_{\|} / E_{\text {run }}$, such as that given by Equation (11).

Thus, to explain the transition from the case of NP 0532 to that of PSR 0833, we assume that for the latter star, because of its initial conditions and aging (which implies slowing down of rotation and probably decrease of $T_{e}$ ), the ratio $E_{\|} / E_{\text {run }}$ is no longer sufficient to maintain an adequate population of fast particles and ensure the excitation of high-frequency modes and X-ray and optical emission. As for the emitted spectrum, for instance in the X-ray region, this can be obtained from the evaluation of the quasi-equilibrium distribution function and of the rate of pitch-angle scattering by the quasi-linear theory (Shapiro and Shevchenko, 1968) of the anomalous Doppler effect represented by the resonance (10).

Again for the other regions of emission, optical and radio, it is possible, by evaluating the matrix elements of the various forms of couplings, to arrive at an estimate of the spectrum. This requires a detailed analysis that is in progress, but given the variety of modes which can be excited, there does not seem to be particularly strong difficulties to arrive at a fit of the available experimental data.

\section{High-Energy Particles}

A mechanism for production of highly relativistic particles can be proposed by identifying them with the 'runaway' particles which escape the effects of collisions and of collective phenomena and undergo almost free acceleration along the magnetic field. 
It is important at this point to recall that an electromagnetic wave of frequency $\omega_{0}$ is emitted outside the light speed cylinder from the rotation of a non-axisymmetric magnetic configuration (Deutsch, 1955; Pacini, 1968). This wave tends to sweep all charged particles from the light speed cylinder and our model provides a continuous injection of particles that can be transported, without relevant energy loss, to distances of the order of the Crab Nebula extended X-ray source $\left(\simeq 10^{18} \mathrm{~cm}\right)$. This process involves a very efficient acceleration of the particles, which are forced to remain almost in phase with the electromagnetic wave, as has been checked by numerical computations (Ferrari and Trussoni, 1971).

The resulting high-energy particles can emit synchrotron radiation over a wide area in the nebular magnetic field. The extended X-ray, optical and radio source can, in fact, be the result of such a process.

We also recall that the electromagnetic wave radiation and the related particle acceleration most likely represents the main energy loss of the rotating neutron star. For the Crab pulsar, assuming $B_{\text {surf }} \simeq 10^{12} \mathrm{G}$, this energy loss can amount to $\approx 10^{38} \mathrm{erg} / \mathrm{sec}$ and, with reasonable values of the parameters of the star, the period lengthening of that pulsar can be explained (Pacini, 1968; Gunn and Ostriker, 1969).

\section{X-Stars and Cosmic-ray Sources}

Finally, we point out the possibility of interpreting other X-ray sources in terms of rotating collapsed bodies. Non-thermal emission mechanisms of the type outlined previously could then be applied to the interpretation of point $X$ sources with a non-thermal spectrum. These would differ from NP 0532 mainly by the absence of the regular pulse pattern and of a nebula in the vicinity of the star which make the source pointlike to the observer. The absence of observed pulsed emission can be attributed to a magnetic configuration that is symmetric around the rotation axis, or never points its beams of enhanced emission towards the Earth, while a high steady background exists.

Particles that are able to escape the effects of collisions and plasma collective effects, undergoing almost free acceleration by $E_{\|}$close to the star, and not affected by a nebula will contribute to the high-energy tail of the cosmic-ray spectrum. The combined high values of rotation and magnetic field of a collapsed star can therefore provide a direct mechanism for high-energy particle acceleration, with the consequences that a sizeable cosmic-ray anisotropy may be present at very high energies.

\section{Acknowledgements}

It is a pleasure to thank A. Treves for his criticism and a number of valuable suggestions. One of us (A.F.) wishes to thank for hospitality the Massachusetts Institute of Technology, where this work was started. 


\section{References}

Artsimovich, L. A., Babrovoskii, G. A., Mirnov, S. V., Rasumova, K. A., and Strelkow, V. S.: 1967, Soviet Atomic Energy 22, 325.

Chandrasekhar, S.: 1961, Hydrodynamic and Hydromagnetic Stability, Oxford University Press.

Coppi, B. and Mazzucato, E.: 1971, Phys. Fluids 14, 134.

Coppi, B., Rosenbluth, M. R., and Sudan, R. N.: 1969, Ann. Phys. 555, 207.

Deutsch, A.: 1955, Ann. Astrophys. 18, 1.

Drake, F. D.: 1969, paper presented at the 131st AAS Meeting, New York.

Eastlund, B. J.: 1968, Nature 220, 1293.

Erber, T.: 1966, Rev. Mod. Phys. 38, 626.

Ferrari, A. and Trussoni, E.: 1971, Nuovo Cimento Letters, Serie 2, 1, 137.

Ginzburg, V. L. and Zaitsev: 1969, Nature 222, 230.

Gold, T.: 1968, Nature 218, 713.

Goldreich, P. and Julian, W. H.: 1969, Astrophys. J. 157, 869.

Gurevich, A. V.: 1961, Soviet Phys. JETP 12, 904.

Hillier, R. R., Jackson, W. R., Murray, A., Redfern, R. M., and Sale, R. G.: 1971, Astrophys. J. 162, L177.

Kadomtsev, B. B. and Pogutze, O. P.: 1968, Soviet Phys. JETP 26, 1146.

Kaplan, S. A. and Tsytovich, V. N.: 1969, Soviet Phys. Usp. 12, 42.

Kinzer, R. L., Noggle, R. C., Seeman, N., and Share, G. H.: 1971, Nature 229, 187.

Large, M. I., Vaughan, A. E., and Mills, B. Y.: 1968, Nature 220, 340.

Neugebauer, G., Becklin, E. E., Kristian, J., Leighton, R. B., Snellen, G., and Westphal, J. A.: 1969, Astrophys. J. Letters 156, L115.

Ostriker, J. P. and Gunn, J. E.: 1969, Astrophys. J. 157, 1395.

Pacini, F.: 1968, Nature 219, 145.

Shapiro, V. D. and Shevchenko, V. I.: 1968, Sov. Phys. Usp. 27, 635.

Smith, F. G.: 1970, this symposium, Paper 8.1, p. 431.

Stodiek, W.: 1969, private communication.

Vasseur, J., Paul, J., Parlier, B., Leray, J. T., Forichon, N., Agrinier, B., Boella, G., Maraschi, L., Treves, A., Buccheri, R., and Scarsi, L.: 1970, Nature 226, 536. 